\title{
Urbanheart surgery - A logic of Design Alternatives
}

\author{
Rollo J, Esteban Y \& Smith D \\ School of Architecture and Building Deakin University-ajrollo@deakin.edu.au
}

\begin{abstract}
In 1972 Sir Leslie Martin in his essay "The Grid as Generator", advocated "a strong theoretical basis for [planning and] urban design" (Carolin P, 2000, p4) by methodically shifting design parameters regarding the way "in which buildings [could be] placed on the land" Martin was able to demonstrate how the generation of alternatives could "allow wider scope for decisions and objectives" to be considered and discussed (Carmona M, \& Tiesdell S 2007, p81). Operating within a conventional design studio yet drawing of Sir Leslie Martin's logic, ie developing an informed understanding of a problem by identifying a finite world of design 'alternatives', the following paper outlines a studio based program at the School of Architecture and Building, Deakin University, referred to as the 'UrbanHeart Surgery'. While most atelier-based courses operate largely on an ad-hoc basis where students often work within self imposed competitive isolation, Urbanheart adopts a more open yet structured approach where students work in design collaboratives to generate a matrix of alternative design scenarios. The program actively integrates postgraduate students from Architecture, Urban Design and Planning into a design research culture and allows them to engage in critical discourse by working on strategic design projects in three areas significant to the future development of the state of Victoria: Metropolitan Urbanism, Urbanism on the Periphery and Regional Urbanism.
\end{abstract}

Keywords: Urban design, alternatives, planning process, design as research 


\section{Introduction:}

UrbanHeart is an urban design forum that integrates students entering their second-degree program into a studio based design research culture and allows them to engage in critical discourse by working on high profile strategic design projects in the state of Victoria. With a population of 4.6 million people, of which over one third, 1.3 million (DSE 2003), reside in rural areas and regional centers outside of the greater Melbourne metropolitan area, the design forum cycles an annual workshop program on three areas significant to Victoria's future development: Metropolitan Urbanism, Urbanism on the Periphery; and Regional Urbanism.

\section{UrbanHeart:}

Working with contemporary, regional and metropolitan issues, the aim of the program is to identify and analyze various factors that make up a precinct's existing conditions, and to develop a range of generic planning strategies and design proposals that address a set of predetermined issues and parameters. Developed with local planning authorities prior to the commencement of the trimester, these issues often involve: the consolidation of suburban sprawl, the resolution of areas of discontinuity, or the development of options for stimulating urban renewal (NB Since the amalgamation of many smaller authorities in the early 1990s and the need to re-assess the urban-seams of past political boundaries, the second issue is often a key focus for many local governments). With regards to predetermined parameters, the constraints considered usually involve demographic, and socioeconomic issues, such as significant versus limited population growth, or diverse versus narrow socioeconomic profile. UrbanHeart introduces seven objectives to the students at the start of the trimester:

a) Develop an understanding of the place of Architecture within the context of the city and become familiar with the preparation of an 'Urban Design Framework' (UDF)

b) Assist local governments in anticipating change and enhancing the (UDF) brief development process by presenting the studio as a platform for explorative strategic thinking.

c) Engender a sense of social value and receptiveness through the community consultation process.

d) Understand the current and future requirements of an integrated urban culture and the significance of the regional city in the state of Victoria.

e) Establish a forum that breaks down piece-meal development between neighbouring municipalities and to facilitate a landscape of decision-making that stimulates an integrated approach to design within the urban context.

f) Enhance community awareness of the architectural profession

g) Become familiar with the Building and Construction Industries current action agenda by developing architecture and building through collaborative and inter-disciplinary design/development initiatives

Students are first introduced to urban design as being a multi-disciplinary process involving a wide range of stakeholders both within a municipality and across adjacent precincts. They are also made aware that it not only involves thinking about opportunities and visions at all scales, but in particular, their implications and consequences both now and long term. By treating study areas with varying levels of objectivity the aim of the forum is not to resolve a 'finite', 'optimum' or 'ideal' solution, but, to collectively identify a broad range of opportunities and design worlds.

Since its introduction in 1999, the forum has developed into a successful teaching, research and public/community relations program. It has not only secured an ongoing relationship with various planning authorities, but its core of industrial partnerships has expanded to include four regional councils (Bendigo, Ballarat, Geelong and Warrnambool), three metropolitan municipalities (Melbourne City, Port Phillip and Wyndham) and close links with various branches of the state government.

The program receives significant media coverage on a regular basis, exposure to a large number of building developers and architectural firms, and students have had the opportunity to present their strategies to state government at a ministerial level on a number of occasions.

\section{Teaching and Delivery of the Unit}

In 1972 Sir Leslie Martin in his essay "The Grid as Generator", advocated "a strong theoretical basis for [planning and] urban design". When it was published, Martin's paper provided a fresh perspective to the notion of formal planning and design processes which had been seriously been challenged by Jan Jacobs with her work 'The Death and Life of Great American Cities'(1961) and Christopher Alexander's 'A City is not a Tree' (1966). Methodically shifting parameters, Sir Leslie Martin adapted Frensel's square diagram as an example of how to generate eight 'alternatives' regarding the way "in which buildings [could be] placed on the land". The diagram 
illustrates nine successive annular rings diminishing in width yet preserving the same area of its predecessor. Martin's rational followed that while the different permutations probably wouldn't have been considered through an informal means of designing, they nonetheless "allow wider scope for decisions and objectives" to be considered and discussed (p81), hence promoting discussion about the complications and implications of each morphological structure.

Involving representatives from the profession, state government and local planning authorities in a conference style method of course delivery, students are provided with a large body of knowledge at the early stages of the program. Students conduct their project work in teams of three, referred to as design collaboratives. Following a two-week period of preliminary research, involving site/precinct investigation, context analysis and SWOT assessment, tutorial groups generate a large range of ideas and "what if?" scenarios within a think-tank environment. Working within a set of constructs, such as socio economic and demographic projections, or public and private space, ideas are pooled across the whole studio and classed within a matrix of permutations (Figure 1). Each design collaborative adopts one of the permutations and resolves their respective strategy into a highly developed proposal (Figure $2 \mathbf{a} \& \mathbf{2 b}$ ).

Land Use: Public / Private Realm

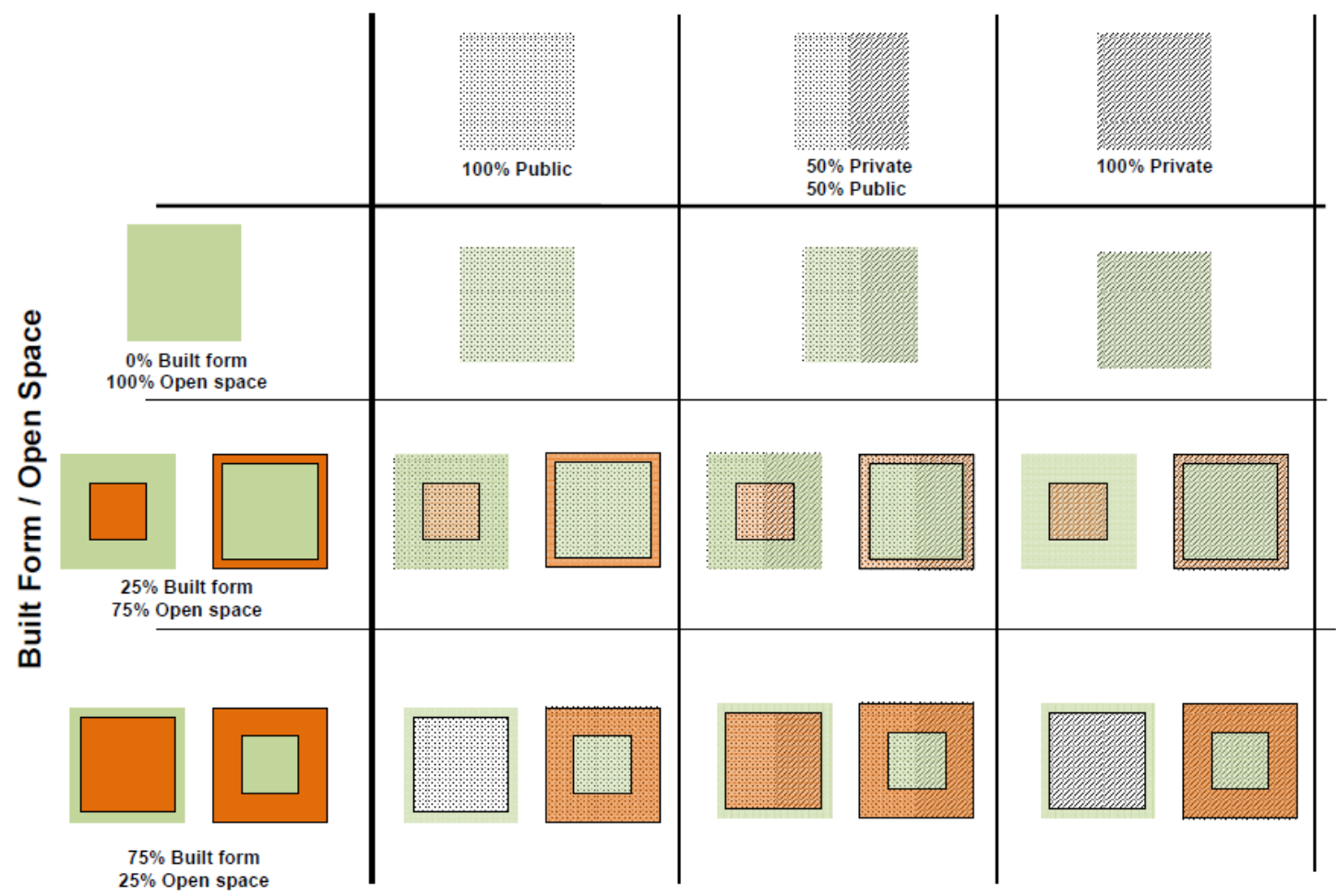

Figure 1 An example of a design matrix illustrating generic alternatives form a correlation public and private land-use with built-form and open space. While the matrix provides a landscape of hybrid combinations, each combination actually holds an extensive array of urban design outcomes . 


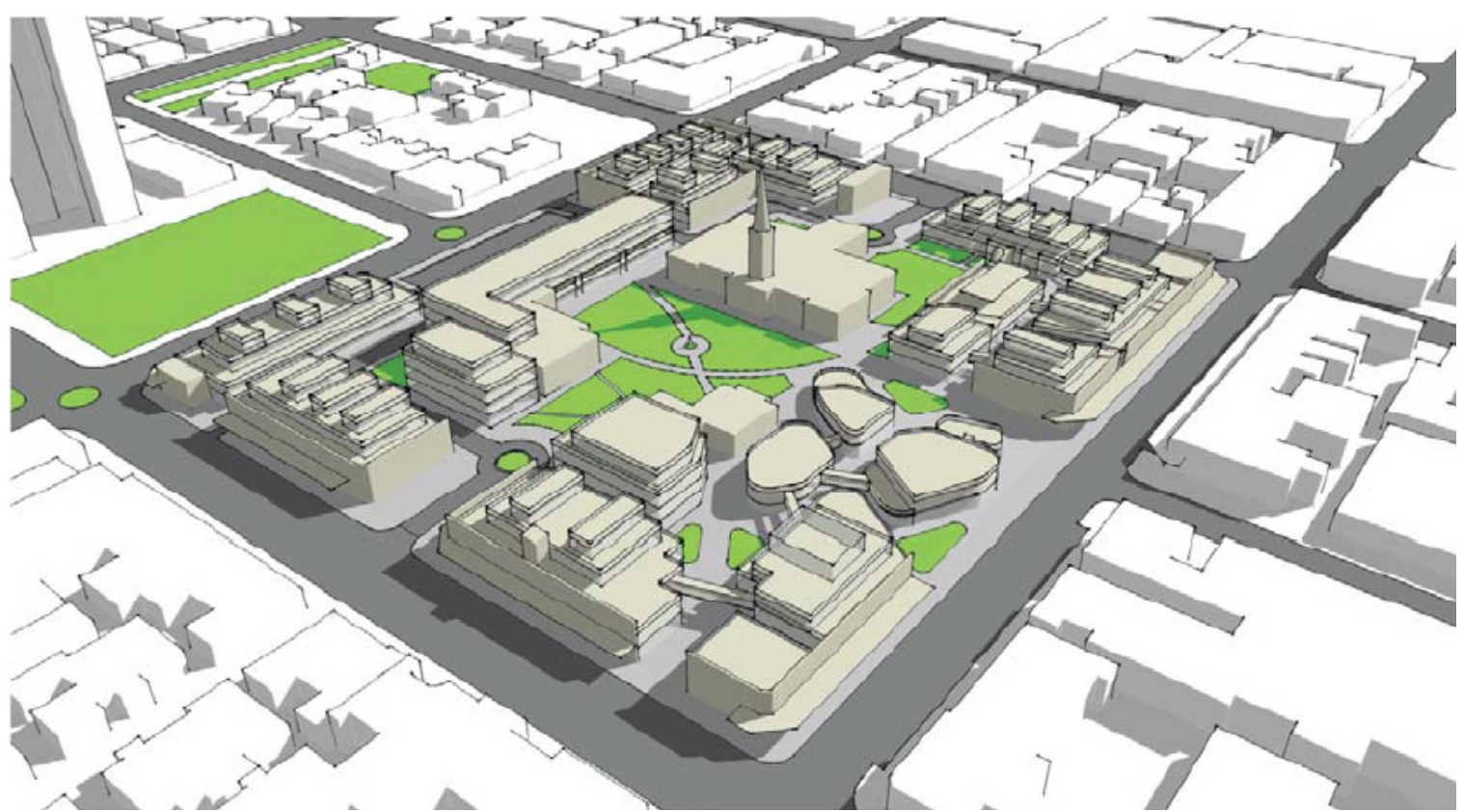

Figure 2a Leong, C.K., Xu, R., Cheol, W.Y. (2009) Revisioning Emerald Hill

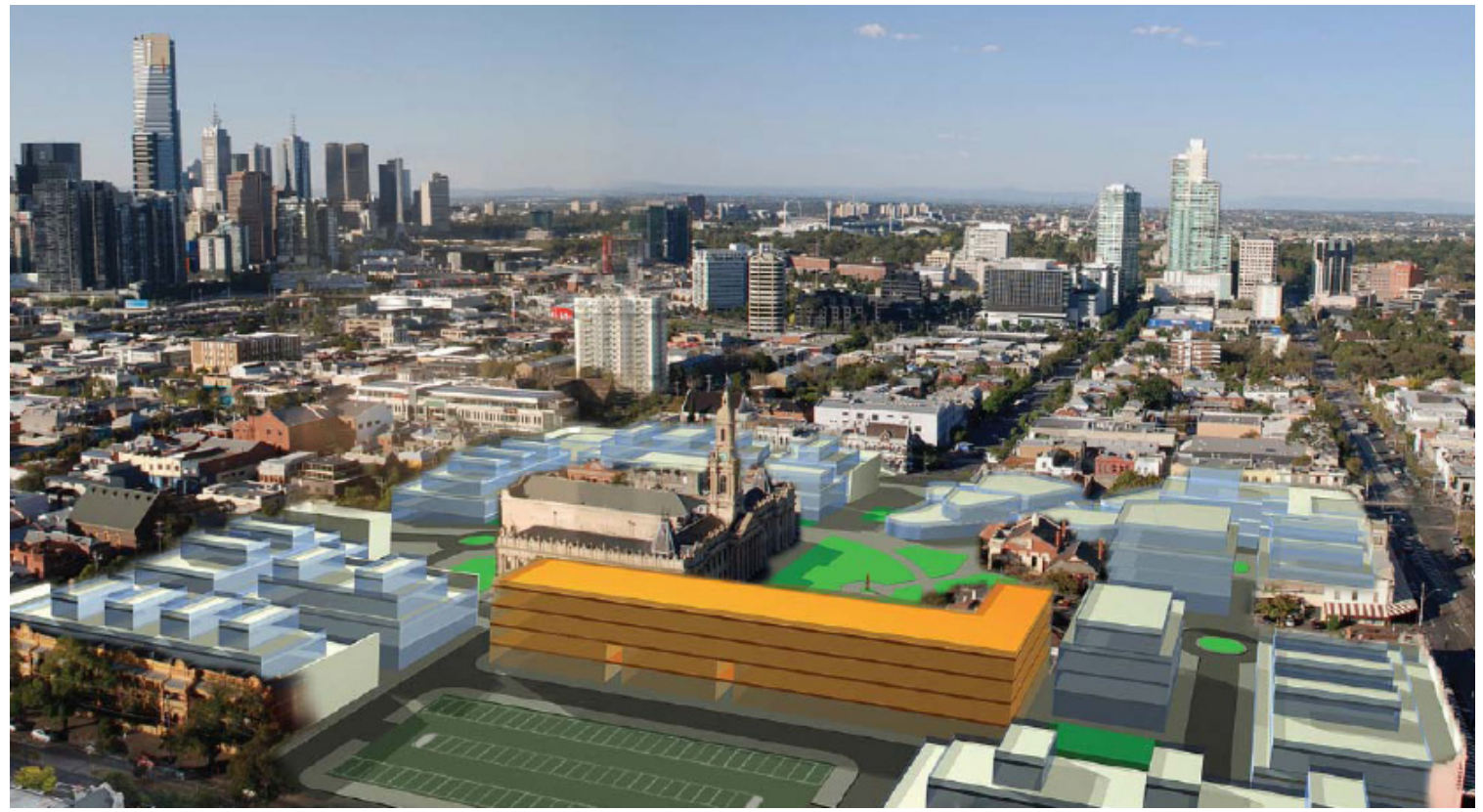

Figure 2b Leong, C.K., Xu, R., Cheol, W.Y. (2009) Revisioning Emerald Hill.

The research forum differs from a conventional studio in both program structure and method of tutoring. Many conventional studios operate largely on an ad-hoc principal. While students are allowed to purse individual initiatives irrespective of their peers, convergence of ideas within a conventional cohort often leaves significant gaps in alternative design solutions. UrbanHeart attempts to counter this deficiency by introducing students to a logic of 'design worlds'. By investigating a large range of design options and, understanding the implications and consequences of such options, all students become familiar with a more extensive set of outcomes and problem solving abilities.

The work developed in each research forum is digitally captured and deposited in an on-line web based repository. Operating as a teaching, research and public interface resource, the repository is utilized by students, the community, and state and local government planning authorities to assist in broadening discussion regarding the possible implications and consequences of forecasted demographic change

\section{Taking architecture and urban design to the community}


The ability of Local governments to explore strategic planning options is severely limited in time, money, and resources. In the process of attempting to help fill this gap, UrbanHeart has developed into a platform where metropolitan and regional municipalities are, for a brief moment, able to engage in positive discourse between members of the architectural profession and construction industry; local communities; and both state and local government. This is executed at two levels: first within the studio through interim and final review sessions; and via the installation of a number of public exhibitions.

The composition of each review panel consists of a mix of discipline groups, including: architects, planners, councillors, landscape designers and community representatives. With the students and their proposals acting as facilitators, the review sessions become a two-way feedback and critique process. While students are able to gauge the competence of their work, many of the proposals act as yard-stick for the review panels to re-assess municipal and council objectives. In order to facilitate strong discourse within cross-discipline juries, all submissions consist of: pre and post scenario analytical diagrams; solid and digitally enhanced models; sketch overlays, photomontages, and collages and serial vision sequences.

Often enhancing a community consultation process, a selection of project work is exhibited in a local gallery or town hall at the conclusion of the studio. The exhibitions inform the public with regards to the vital role which architects can play in moulding their built environment and councils are able to utilise the work in testing community response to both the type and degree of change which their community is willing to embrace.

\section{Contribution to Research}

Research outcomes directly relating to the studio operate on two levels, none-funded and funded. The nonfunded initiatives are, by and large, the direct result of the studio program. When presented in a format that addresses a series of problems within a given set of parameters, the projects stand as body of sound investigative work. This can be disseminated though hard copy journal publications, public exhibitions or online repositories, and can also act as a primer for further investigation by graduate research students at honours and masters levels.

By imposing a comparatively 'clinical' approach to the studio, and directly tying the project work with the strategic planning objectives of local governments, critical discourse, resulting from the review sessions and community feedback, often leads to the distillation of more generic issues. These are either current, or forecasted to impact on the community and profession in the mid to long-term future. Hence problems, relating to questions of demography, future infrastructure needs, and the quality of the built environment become the drivers of the UrbanHeart design research group in forming partnerships with other professional and community based organizations (Figures $3 \& 4$ ). These partnerships inturn become the basis on which we are able to facilitate specialised funded research with organisations such as the Australian Research Council.

\section{Conclusion}

While many tutors prefer an ad hoc and loose approach to studio teaching/supervision as opposed to a more collaborative and directed structure: the vision-benefits to local government authorities; the applied teaching benefits to students who take part in the development of a diverse range of proposals; and the research outcomes from addressing current and forecasted urban issues, are significant.

It would probably be inappropriate to activate all studios along such lines. Yet, introducing students in the early stages of the upper degree to a design research culture based on exploratory investigative principles and critical discourse, appears to offer a range of generic skills for developing a more informed and "definitively postgraduate” design based intuition. 

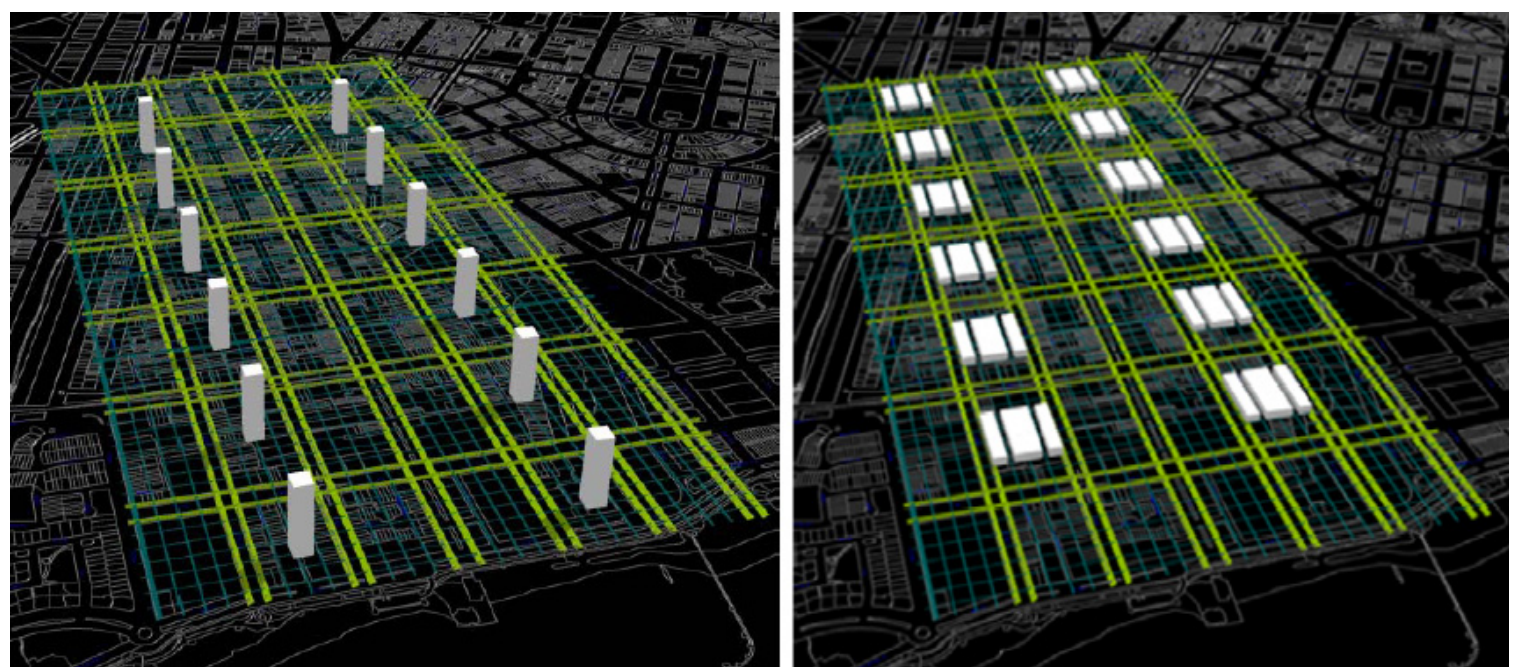

Figure 3 - 3D Model - Modelling future growth scenarios, built form permutations - Port Melbourne Activity Centre $100 \mathrm{~m}^{2}$ apartments - building height 4 storey and 20 storey (Y Esteban)

Scenario based demand assumptions:

1) Estimated 1,926 additional dwellings modeled across the suburb by 2030 .

2) Estimated 963 dwellings modelled within 400m of a Major Activity Centre.

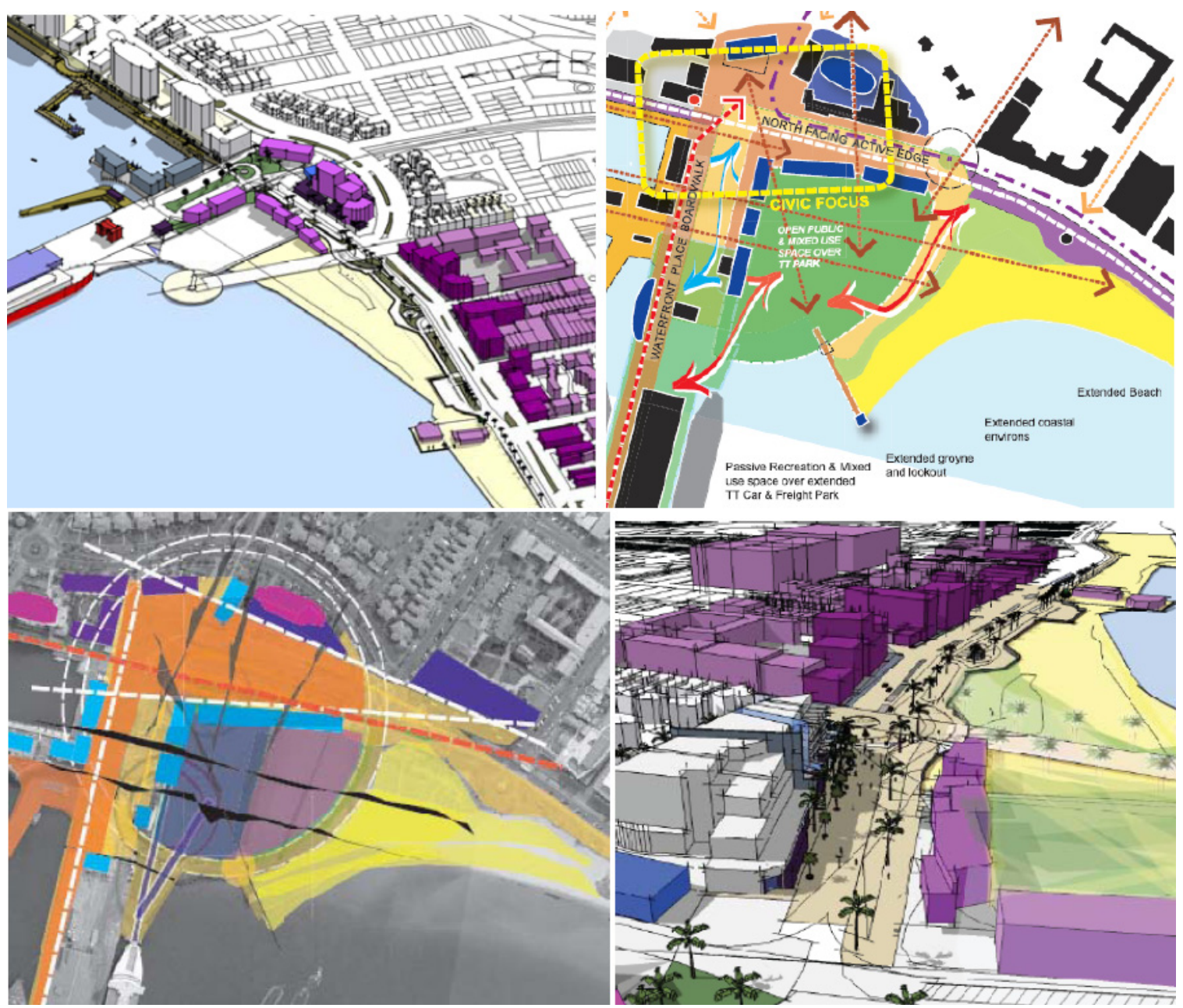

Figure 4 - Port Melbourne Waterfront Revitalisation - Integrated Vision (Urbanheart 2007)

Public Exhibitions: http://www.urbanheart.net 
Design Research Forums and exhibitions to date include:

Regional Urbanism / Warrnambool Heart Beats - Warrnambool 2003; Port Melbourne Foreshore - City of Port Phillip 2003; Shakespear Grove and West Beach - City of Port Phillip 2003; Urban Villages / Urban Injections - City of Port Phillip 2002; Regional Urbanism / Ballarat Heart Beats - Ballarat 2002; Western Wedge Geelong 2002; Crossing the Political Boundary - Melbourne City \& Port Phillip 2001; Urbanism on the Periphery / Drawing on Werribee - Wyndham 2001; Eco Island - City of Port Phillip 2000; Finding the Pulse - Bendigo 2000; Urban Heart Surgery -Uncharted Territories Unlimited Horizons - Port Phillip 1999.

\section{Bibliography}

Carolin P. Obituary, Leslie Martin 1908-2000, Architecture Research Quarterly, Vol 4 No 4, 2000

Carolin P. Obituary, Leslie Martin 1908-2000, Martin Centre for Architecture and Urban Studies, Report 2000-2001, P4, University of Cambridge Department of Architecture,

Department of Sustainability and the Environment, State Government of Victoria. http://www.dpi.vic.gov.au

Martin. L (1972) The Grid as Generator, Ed. Carmona M, \& Tiesdell S. Urban Design Reader (p.70) Architectural Press, Elsevier, 2007

Rollo J (November 2002) ECO-ISLAND: Urban Heart Surgery 2002 Uncharted

Territory and Unlimited Horizons. Architect Victoria (November Ed. Rollo J, Veron D)

\section{Biography:}

John Rollo is a senior lecturer with the School of Architecture and Building at Deakin University. He initiated 'Urbanheart Surgery' in 1999, and currently coordinates both the Bachelor of Design (Architecture) and the Masters of Urban Design courses.

Yolanda Esteban is an urban designer and lecturer in Architecture at Deakin University. She holds a degree in both Architecture and Construction Management. Yolanda has worked extensively in the fields of urban design and planning and is a foundation member of the Urbanheart Design Research Group. She is actively involved in the research areas of sustainable urban growth policy and public open space evaluation.

Both authors have worked extensively with Urbanheart, which advances a local/global sustainable healthy cities agenda through a studio-based design research culture, and have facilitated over twelve design research forums for six separate municipalities in both regional Victoria and Melbourne.

Des Smith is Professor of Architecture with the School of Architecture and Building at Deakin University. He has been a practicing architect in Victoria since 1981, and in Western Australia since 1984, and in Western Australia since 1984. He has been lucky enough to have been closely associated with the realisation of over seventy architectural projects in both Victoria and Western Australia. These projects include residential, commercial and public buildings. He has also been closely associated with architectural projects for Indigenous Communities in Western Australia (in the Kimberly and the Wheatbelt Regions of that vast state). 